\title{
Acquisition of quantifier raising of a universal across an existential: Evidence from German
}

Kriszta Szendrői, Rebecca Schumacher, Tom Fritzsche \& Barbara Höhle

\section{Appendix 1}

\section{Output of the statistical model:}

- Agegroup is coded such that 6yo are in the intercept - so that they can be compared to 5 yo and adults.

- Condition is coded such that active (= experimental items) are in the intercept.

- The model has three random components: for participants, for items, and an individual adjustment of the condition effect for each participant (= random slope).

- The estimates are in $\log$ odds (zero $=1: 1=50 \%$ ).

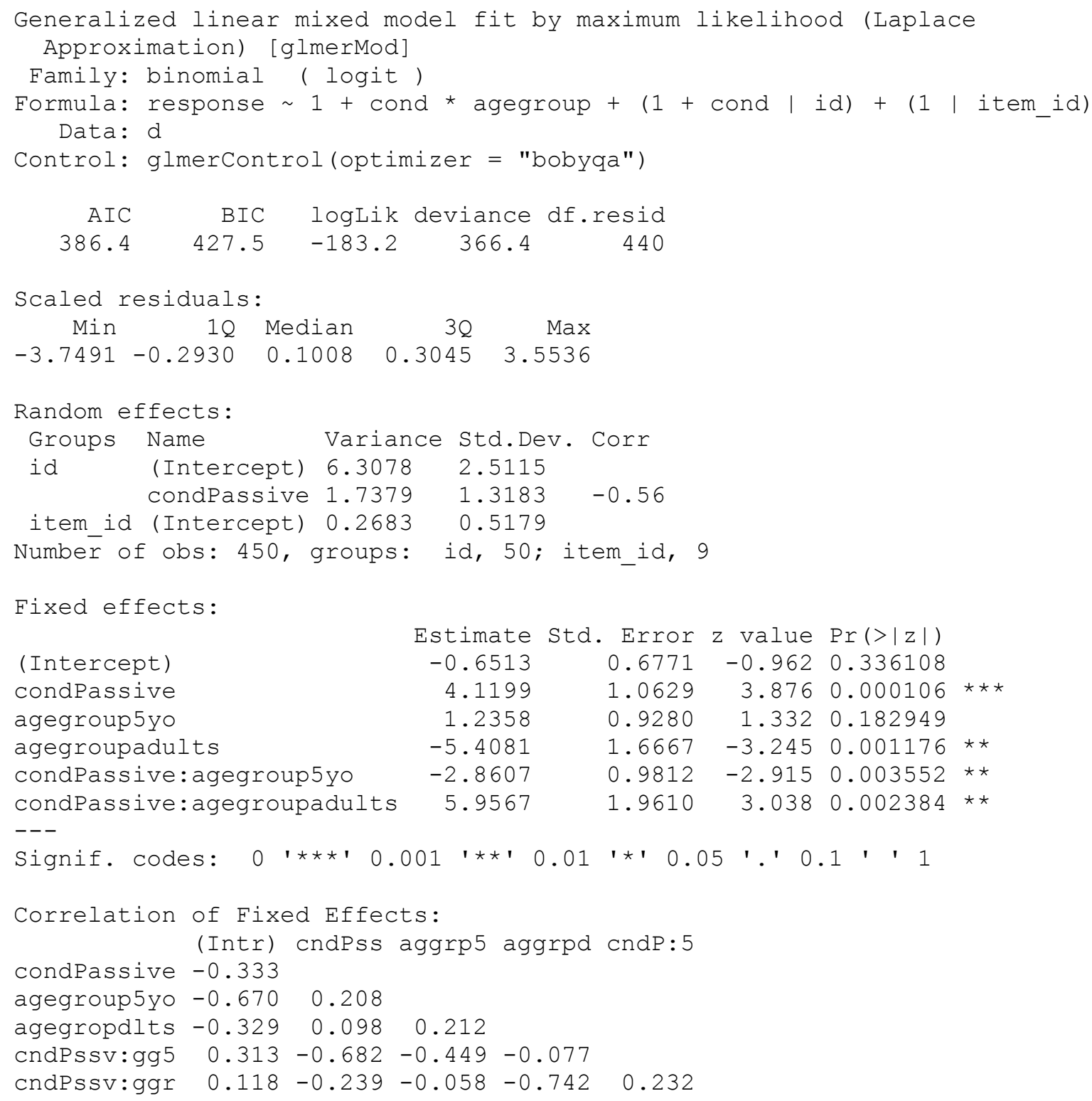

\section{Explanation of the fixed effects:}

(Intercept)

- This is the average of the 6yo in the actives (in log odds, the $42 \%$ in the plot, o would be $50 \%$ ). 
- All other effects are added to this (so are in relation, meaning they are not absolute effects like main effects in ANOVA).

condPassive

- The effect of passives compared to actives for 6yo, so the increase to 90\% ( $\mathrm{p}<.001)$.

- 6yo give more distributive responses in passives than in actives.

agegroup5yo

- The difference between $5 y 0$ and $6 y 0$ for the actives $(p=.183)$.

- The value is positive, which means that 5yo give numerically more distributive responses to actives than 6yo do, but this is not significant.

agegroupadults

- The difference between adults and 6yo for the actives $(\mathrm{p}<.01)$.

- Adults give significantly less distributive responses (2\%) to actives than 6yo do (42\%).

condPassive:agegroup5yo

- The difference between the passive effect for 5 yo $(+17 \%)$ and the passive effect for $6 y o(+48 \%$ increase).

- The size of the passive effect is reduced by $31 \%$ in 5 yo compared to $6 y 0(p<.01)$.

condPassive:agegroup6yo

- The difference between the passive effect for adults (+91\%) compared to 6yo $(+48)$.

- The passive effect is significantly larger (by $43 \%)$ in adults compared to the one in 6yo ( $\mathrm{p}<.01)$.

- The effect of passive is significant in 6yo and even larger in adults.

The only comparison that this model does not give is whether the difference between $56 \%$ for actives compared to $73 \%$ for passives in 5 yo is significant. To test this, the 5 yo have to be put in the intercept (see model 2 below). 


\section{Appendix 2}

\section{Output of model 2:}

- The model specification is identical to the first model, just the reference level for the factor agegroup was changed from 6-year olds to 5 -year olds.

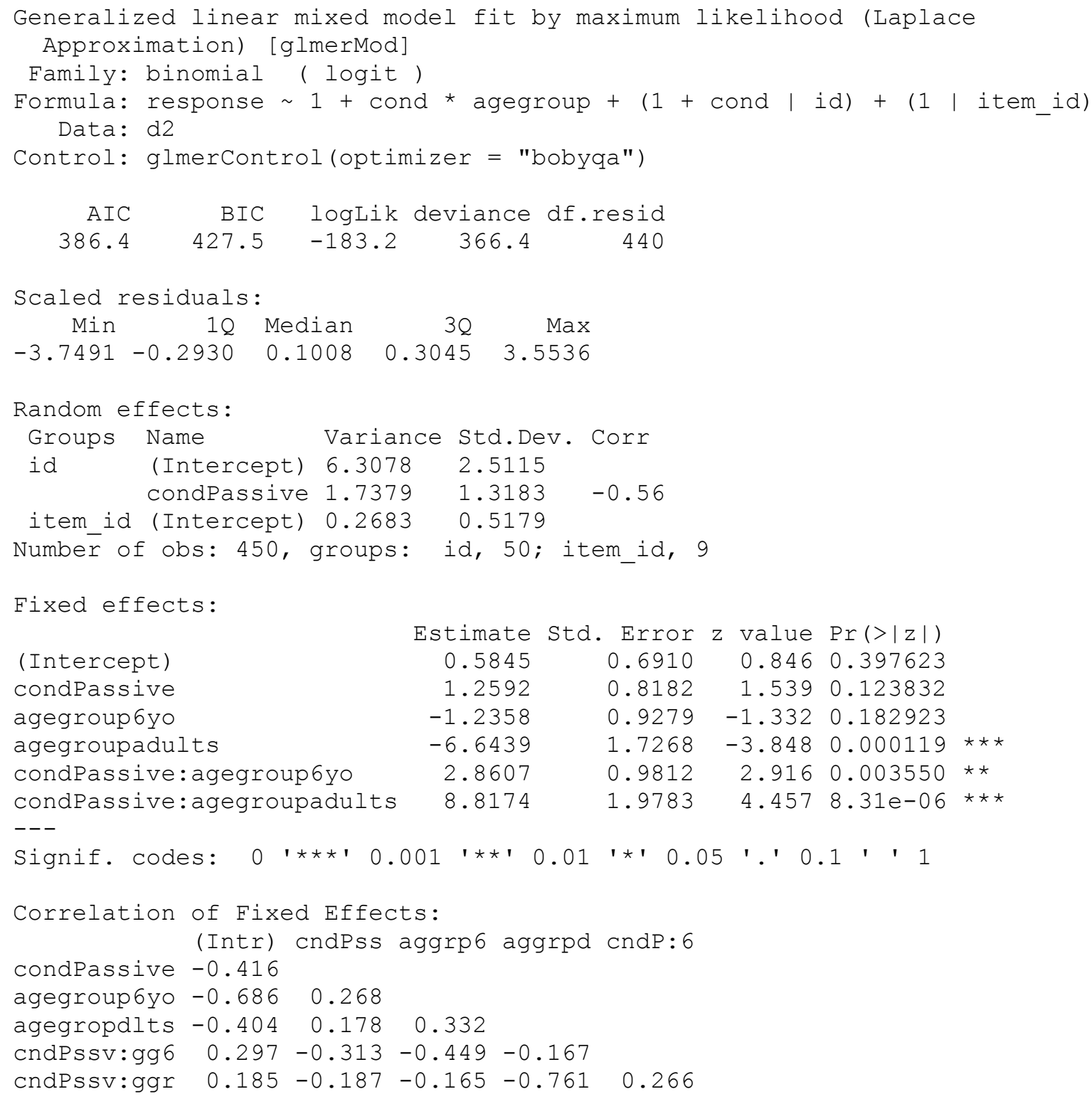

Relevant here is the condPassive effect, which now refers to only the 5yo:

condPassive

- The (absolute) effect of passives for $5 y 0$ ( $1.26=$ the $17 \%$ increase) is not significant $(p=.124)$. The first model above already showed that the 5 yo differ from $6 y o$ for this effect, now this model shows that there is no significant effect of passives in 5 yo.

- The rest of the model is very similar (regarding the significance level: identical) to the model above, the estimates change a bit because now it is in relation to the 5yo. But note that the effect agegroup6yo is identical to the effect agegroup5yo above just the sign of the estimate changes because now 6yo are compared to 5yo, before it was the other way around. 